\title{
Characteristics of optical limiting in media with nonlinear absorption and scattering
}

\author{
S.E. Zelensky, O.S. Kolesnik, O.V. Kopyshinsky \\ Taras Shevchenko Kyiv National University, Physics Department \\ 6, prospect Glushkova, 03680 Kyiv, Ukraine E-mail: zele@univ.kiev.ua
}

\begin{abstract}
A comparative study is performed for characteristics of optical limiting in the media with nonlinear absorption and scattering with the use of nanosecond-scale laser pulses. Two methods are proposed to analyze of experimental nonlinear transmittance curves. The experiments revealed differences in the shape of nonlinear transmittance curves in the media with different physical mechanisms of optical limiting. For optical limiting in suspensions of light-absorbing particles, it is concluded that the fifth-order nonlinear susceptibilities should be taken into account.
\end{abstract}

Keywords: optical limiting, laser-induced absorption, nonlinear scattering, nonlinear transmittance curves, carbon black suspensions.

Manuscript received 24.05.05; accepted for publication 25.10.05.

\section{Introduction}

One of the effects of self-action of laser beams propagating through nonlinear media is the dependence of optical transmittance (or the extinction coefficient) on the laser intensity. Several physical mechanisms are known to be responsible for laser-induced changes of optical transmittance at high levels of the laser power. For example, saturated absorption in atoms and molecules due to the decrease of occupance of lower energy levels is the well-known phenomenon that is widely used in quantum electronics and laser spectroscopy [1, 2]. Similar behavior of transmittance curves is observed in the case of non-stationary selfinduced transparency $[1,2]$, however, its non-stationary physical mechanism differs from the mechanism of simple saturated absorption. Besides, the enhancement of optical transmittance is observed for powerful laser beams interacting with aerosols [3].

It is not a rare occasion when a laser pulse of a moderate power density demonstrates power-dependent self-induced attenuation during propagation through various media. In these cases, the laser-induced decrease of optical transmittance is observed, i.e. the nonlinear medium tends to limit the transmitted laser power. Such effects are called optical limiting that has promising applications in the field of laser safety of photodetectors, etc.

One of the mechanisms of optical limiting is the laser-induced absorption (excited-state absorption of atoms and molecules, absorption by photochemical products, absorption by laser-ionized atoms, molecules, impurity centers, etc.) [4-10].
Interaction of powerful laser pulses with suspensions of light-absorbing submicron and nano-size particles (carbon black suspensions, carbon nanotube suspensions, etc.) demonstrates effective broadband optical limiting [11-15]. Unlike the nonlinear absorption, the primary mechanism of optical limiting in such colloidal media is the laser-induced nonlinear light scattering, i.e., the increase of scattering cross-section with the increase of the laser power.

It should be also mentioned that the absence of laserinduced changes of optical transmittance does not grant the absence of non-linearity in the processes being investigated. For example, when the cross-sections of linear and non-linear absorption coincide, the experiments show the laser power-independent optical transmittance accompanied by essentially nonlinear luminescence (see, for example, [16]).

Self-induced changes of optical transmittance of laser beams can be treated as non-coherent nonlinear optical phenomena by using nonlinear susceptibilities of odd orders, $\chi^{(2 n-1)}$ [2]. For example, to a first approximation, in a great number of instances, laserinduced absorption can be interpreted by the presence of the non-zero imaginary part of the third-order susceptibility tensor, $\operatorname{Im} \chi^{(3)}(\omega=\omega-\omega+\omega) \neq 0$. It should be noted that the phenomena of nonlinear absorption can be treated using the microscopic models accounting for optical transitions in atoms and molecules, without the use of formalism of optical susceptibilities.

In the present paper, we perform a comparative study of peculiarities of optical limiting of nanosecond-scale 
laser pulses in different media with excited state absorption and with nonlinear scattering. It is shown that the analysis of the shape of nonlinear transmittance curves provides useful information about the physical mechanisms of powerful laser radiation interaction with matter. We propose two methods to analyze the shape of nonlinear transmittance curves. These methods enabled us to reveal the nonlinearities of high orders (fifth and higher) in the experimental transmittance data.

\section{Experimental details}

The experiments were performed using the harmonics of Q-switched YAG: $\mathrm{Nd}^{3+}$-lasers $\quad(1064 \mathrm{~nm}, 355 \mathrm{~nm}$, $266 \mathrm{~nm}$ ) with pulse duration from 10 to $20 \mathrm{~ns}$ in different experiments, with the pulse repetition rate of $(0.5 \ldots 2) \mathrm{s}^{-1}$. Each laser pulse was processed separately; the data averaging was implemented into the software. The laser beams had smooth bell-shaped cross-beam distributions of the power density. Optical transmittance was measured as a ratio of energies of incident and transmitted laser pulses. All measurements were carried out at the room temperature.

The investigations were performed using the following samples. (i) $\mathrm{Y}_{3} \mathrm{Al}_{5} \mathrm{O}_{12}$ garnet crystals doped with $\mathrm{Nd}^{3+}$ ions. A segment of standard laser rod with a thickness of $d=5 \mathrm{~mm}$ was used. (ii) Borate glasses of the following formula $\mathrm{K}_{2} \mathrm{O} \cdot 6 \mathrm{~B}_{2} \mathrm{O}_{3}-0.1 \%$ wt. $\mathrm{SnO}$. The glasses were melted in platinum crucibles in air at the temperature of $\sim 1300 \mathrm{~K}$. (iii) Aqueous suspensions of submicron light-absorbing particles. Diluted and filtered gouache paint was used. A nephelometric estimation of the mean particle size is $0.1 \mu \mathrm{m}$. To eliminate errors caused by laser-induced fading, the investigated suspensions were pumped through the optical cell in such a manner that each laser pulse interacted with a fresh portion of suspension.

\section{Results and discussion}

Suppose the intensity (the surface power density, $F$ ) of laser radiation propagating through a nonlinear medium satisfy the following equation

$$
d F=-\alpha(F) F N d z
$$

where $\alpha$ is the extinction cross section which depends on the laser intensity, $N$ is the concentration of absorption (scattering) centers, $\mathrm{z}$ is the coordinate along the laser beam. To a first approximation, consider the interaction of laser radiation with a medium that can be characterized by the third-order optical nonlinearity (i.e. the laser-induced dipole momentum can be expressed as $\left.\vec{P}=\chi^{(1)} \cdot \vec{E}+\chi^{(3)}: \vec{E} \vec{E} \vec{E}\right)$. As is known for a third-order nonlinear media, the extinction cross section can be presented as a linear function of laser intensity as follows

$$
\alpha(F)=\alpha_{0}+\beta F
$$

where $\alpha_{0}$ is the linear cross section, $\beta$ is the coefficient proportional to the imaginary part of the third-order optical susceptibility, $\operatorname{Im} \chi^{(3)}$.

Denote the surface density of the incident $(z=0)$ and transmitted $(z=d)$ laser powers as $F_{0}, F_{d}$. For a sample thickness $d$, Eqs (1) and (2) yield the following expression for the laser power dependency of the optical transmittance

$T\left(F_{0}\right)=\frac{F_{d}\left(F_{0}\right)}{F_{0}}=T_{0}\left[1+\frac{\beta}{\alpha_{0}}\left(1-T_{0}\right) F_{0}\right]^{-1}$

where the low-signal transmittance, $T_{0}$, satisfies Bouguer's law, $T_{0}=\exp \left(-\alpha_{0} N d\right)$.

Expression (3) shows that the transmitted laser power, $F_{d}$, demonstrates nonlinear response on variations of the incident laser power, $F_{0}$. For further analysis, we introduce the following parameter of nonlinearity

$\gamma_{F}=\frac{d F_{d} / F_{d}}{d F_{0} / F_{0}}$

The dimensionless parameter $\gamma_{F}$ can be easily calculated from the experimental data as a local slope of $F_{d}\left(F_{0}\right)$ curve plotted in a log-log scale. In the case of linear absorption, the transmittance is a constant, and the transmitted power, $F_{d}$, is proportional to the incident power, $F_{0}$, hence the parameter of nonlinearity equals to unity, $\gamma_{F}=1$. As far as $F_{d}=T F_{0}$, obviously, the value of $\gamma_{F}$ can be determined via numerical differentiation of the experimental curves $F_{d}\left(F_{0}\right)$ or $T\left(F_{0}\right)$ as follows

$\gamma_{F}=\frac{d T / T}{d F_{0} / F_{0}}+1=\gamma_{T}+1$

According to the definition (4), the expression (3) yields

$\gamma_{F}\left(F_{0}\right)=\frac{T\left(F_{0}\right)}{T_{0}}$.

Thus, for media with third-order nonlinearities we conclude that the parameter of nonlinearity, $\gamma_{F}$, and the normalized transmittance, $T / T_{0}$, behave similarly with the increase of the incident laser power, $F_{0}$. The calculated curves $\gamma_{F}\left(F_{0}\right)$ and $T\left(F_{0}\right) T_{0}^{-1}$ coincide with the curve 1 plotted in Fig. 1. The validity of the expression (6) can be easily checked up experimentally. However, before doing so, consider possible causes violating the expression (6).

The expression (6) is derived with the assumption of uniform distribution of the laser intensity across the beam. However, as is known, the neglect of non- 


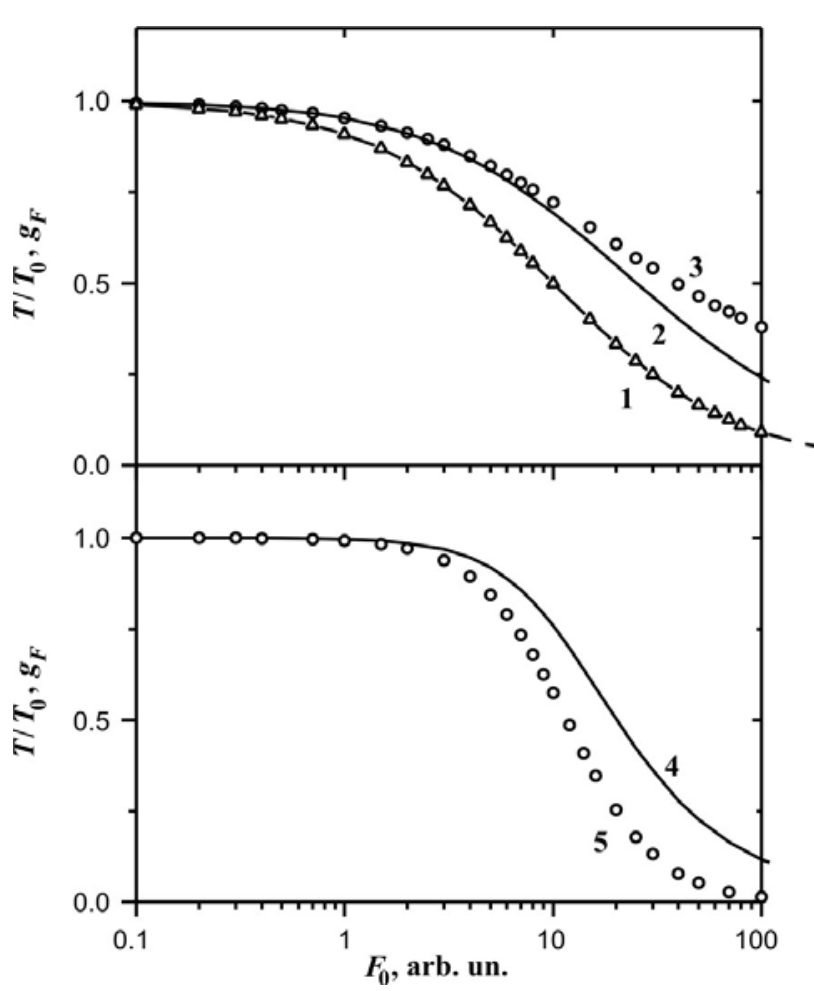

Fig. 1. Normalized transmittance $T / T_{0}$ (solid curves) and parameter $\gamma_{F}$ (points) calculated according to equations (1), (2) (plots 1-3) and (1), (7) (plots 4, 5) for uniform $(1,4,5)$ and Gauss $(2,3)$ cross-beam distribution of laser intensity.

uniformity of laser power cross-beam distribution causes significant errors in nonlinear laser spectroscopy [17, 18]. To check the validity of the expression (6) with non-uniform laser beams, a computer simulation seems to be suitable. Numerical calculations were performed for the $\gamma_{F}\left(F_{0}\right)$ and $T\left(F_{0}\right) T_{0}^{-1}$ dependencies with the Gauss cross-beam distribution of the laser power. The results of calculations are given in Fig. 1, curves 2 and 3. As is seen from the figure, in the region of small changes of the transmittance $\left(T / T_{0}>0.7\right)$, the difference between the curves $\gamma_{F}\left(F_{0}\right)$ and $T\left(F_{0}\right) T_{0}^{-1}$ does not exceed uncertainties of typical pulsed laser experiments, whereas at high levels of the laser power the mentioned curves differ significantly. Therefore, the expression (6) can be applied for analysis at low levels of optical limiting (with keeping the approximate condition $T / T_{0}>0.7$ ).

Consider several examples of media with optical limiting at moderate levels of the pulsed laser power (below $100 \mathrm{MW} \cdot \mathrm{cm}^{-2}$ ). The first example - YAG: $\mathrm{Nd}^{3+}$ crystals. Spectral characteristics of $\mathrm{Y}_{3} \mathrm{Al}_{5} \mathrm{O}_{12}: \mathrm{Nd}^{3+}$ crystals in the visible and near UV regions are determined by optical transitions within $4 f^{3}$ configuration of $\mathrm{Nd}^{3+}$ ions $[19,20]$. At the wavelength $355 \mathrm{~nm}$, the absorption of laser radiation provides an occupance of the metastable ${ }^{2} P_{3 / 2}$ level of $\mathrm{Nd}^{3+}$ ion via ${ }^{4} I_{9 / 2} \rightarrow{ }^{2} P_{3 / 2}$ transitions. With the increase of the laser intensity, the occupance of ${ }^{2} P_{3 / 2}$ level increases significantly, hence the transitions from ${ }^{2} P_{3 / 2}$ to high levels of $4 f^{2} 5 d$ configuration become actual [21]. Optical transitions within $f-f$ configuration are forbidden, whereas $f-d$ transitions are allowed and have large oscillator strengths. That is why, the excited state absorption from the ${ }^{2} P_{3 / 2}$ level to the levels of the $4 f^{2} 5 d$ configuration is easily observed experimentally as a nonlinear transmittance. The results of experiments are given in Fig. 2a. As is seen from the figure, the normalized transmittance and the parameter of nonlinearity practically coincide; this fact agrees with the expression (6). Thus, the excited state absorption in $\mathrm{Y}_{3} \mathrm{Al}_{5} \mathrm{O}_{12}: \mathrm{Nd}^{3+}$ crystals can be approximated to the model $\chi^{(3)}=$ const (the expressions (1) and (2)).

As a second example of the media with optical limiting, consider alkali-borate glasses doped with the mercury-like ions $\left(\mathrm{Tl}^{+}, \mathrm{Pb}^{2+}, \mathrm{In}^{+}, \mathrm{Sn}^{2+}\right)$. Optical characteristics of such glasses in UV and visible spectral regions are determined by the dopants $[22,23]$. The mercury-like ions in glasses form wide absorption and luminescence bands. At high levels of UV laser excitation, such glasses demonstrate optical limiting via the mechanisms of excited state absorption and absorption by ionized centers [9, 16, 22-24]. In the present paper, the glass composition was chosen to minimize the laser-induced ionization as compared with other glasses. The results of experiments are given in Fig. 2b. As is seen from the figure, $T / T_{0}$ and $\gamma_{F}$ coincide within the accuracy of measurements, which substantiates the validity of third-order approximation.

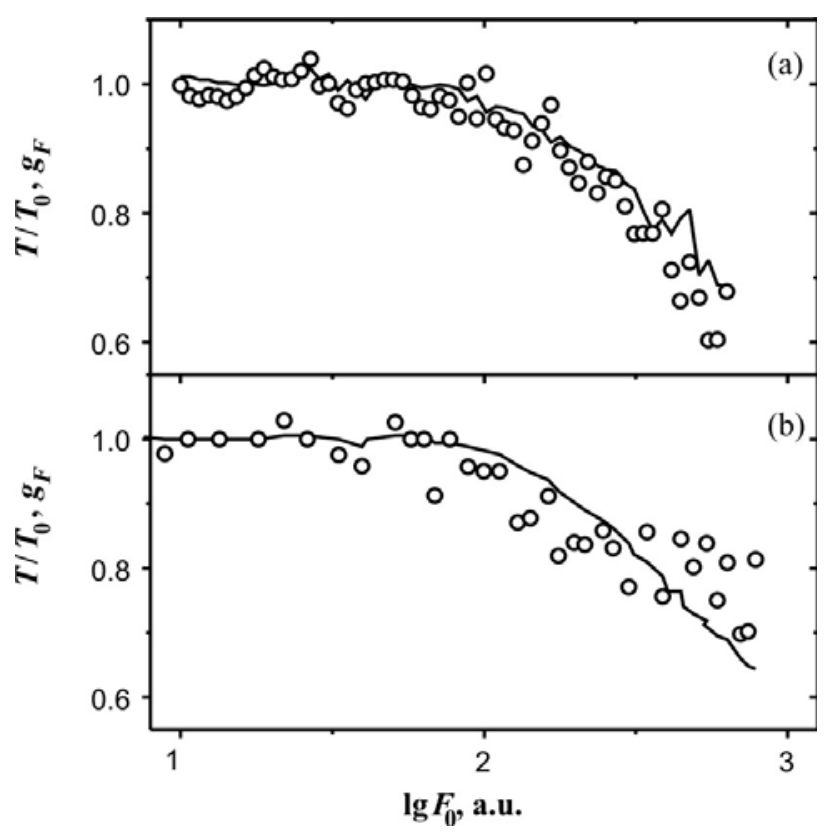

Fig. 2. Normalized transmittance $T / T_{0}$ (solid lines) and parameter $\gamma_{F}$ (circles) in YAG: $\mathrm{Nd}^{3+}$ crystals (a) and in $\mathrm{K}_{2} \mathrm{O} \cdot 6 \mathrm{~B}_{2} \mathrm{O}_{3}-\mathrm{SnO}$ glass (b) as functions of laser power density at wavelengths $355 \mathrm{~nm}$ (a) and $266 \mathrm{~nm}$ (b). 
Besides, it seems surprising, the above-mentioned nonlinear transmittance curves of different objects crystals and glasses - coincide (compare the graphs in Fig. 2a, b).

Finally, consider the third example - aqueous suspensions of black-body particles - where optical limiting is caused by the laser-induced nonlinear scattering. There are two primary mechanisms of the nonlinear scattering in suspensions: (i) vaporization of liquid around the laser-heated suspended particles, and (ii) production of laser plasma in the neighborhood of particles [11-15]. Presently, relative contributions of the mentioned two mechanisms to optical limiting are not completely clear. In $[11,25]$, a simple model is developed for optical limiting in light-absorbing suspensions; the model takes into account laser-induced vaporization of surrounding fluid.

The results of optical limiting experiments with an aqueous suspension are given in Fig. 3. As is seen, the obtained curves $T / T_{0}$ and $\gamma_{F}$ are essentially different: first, $\gamma_{F}$ one rapidly decreases with $F_{0}$ from 1 to $\sim 0.8$, then it slowly decreases to $\sim 0.75$, whereas the decrease of transmittance is smooth. Such behavior of the $T / T_{0}$ and $\gamma_{F}$ dependencies contradicts to the expression (6) and can not be explained within the framework of the assumption of $\chi^{(3)}=$ const .

To a second approximation, assume the investigated medium characterized by nonlinear susceptibilities of the third and fifth orders. Taking account of $\chi^{(5)}$ generates a new summand in the right-hand portion of the equation (1), proportional to the third power of $F$, due to the following expression

$\alpha(F)=\alpha_{0}+\beta F+\mu F^{2}$

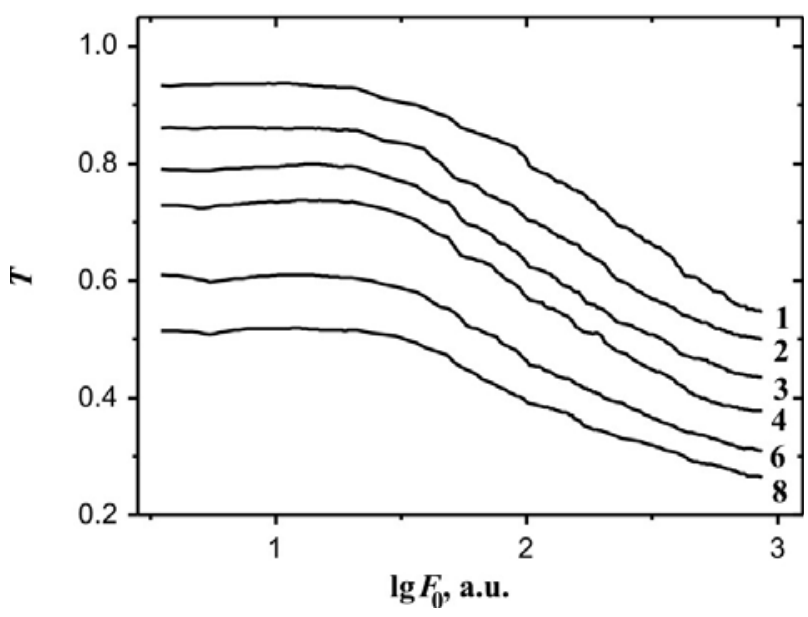

Fig. 3. Normalized transmittance $T / T_{0}(1)$ and parameter $\gamma_{F}(2)$ as functions of laser intensity $F_{0}(1064 \mathrm{~nm}, 20 \mathrm{~ns})$ in aqueous suspension of black-body particles. Low signal transmittance $T_{0} \approx 0.7$. where the coefficient $\mu$ depends on $\chi^{(5)}$. Calculations performed with (1) and (7) showed a significant difference in the laser power dependency of $T / T_{0}$ and $\gamma_{F}$. The curves 4 and 5 in Fig. 1 were calculated for the case of $\beta=0, \mu \neq 0$. As is seen from the figure, taking account of $\chi^{(5)}$ makes the dependency $T\left(F_{0}\right) T_{0}^{-1}$ more abrupt than in the case of $\chi^{(3)}$. Besides, $\gamma_{F}$ decreases with $F_{0}$ more quickly than the transmittance does.

The mentioned peculiarities of $T / T_{0}$ and $\gamma_{F}$ curves calculated in the $\chi^{(5)}$ approximation agree with the results of the experiments given in Fig. 3. In the region of onset of optical limiting, the non-coinciding experimental curves $T / T_{0}$ and $\gamma_{F}$, with $\gamma_{F}$ below $T / T_{0}$, can be considered as a signature of the $\chi^{(5)}$ nonlinearity.

Concerning the role of high-order nonlinearities in the processes of interaction of nanosecond-scale laser pulses with carbon black suspensions, its importance is also confirmed by the results of experiments with degenerate four-wave mixing [26].

It should be noted, application of the considered procedure (the expression (6)) to the experimental data on optical limiting published in [27, 28] gives results similar to those described in the present paper. Namely, optical limiting in the media with nonlinear scattering mechanism (carbon black suspensions, carbon nanotube suspensions) show the signs of $\chi^{(5)}$ nonlinearity, whereas fullerene solutions demonstrate the $\chi^{(3)}$ nonlinearity caused by the excited state absorption mechanism.

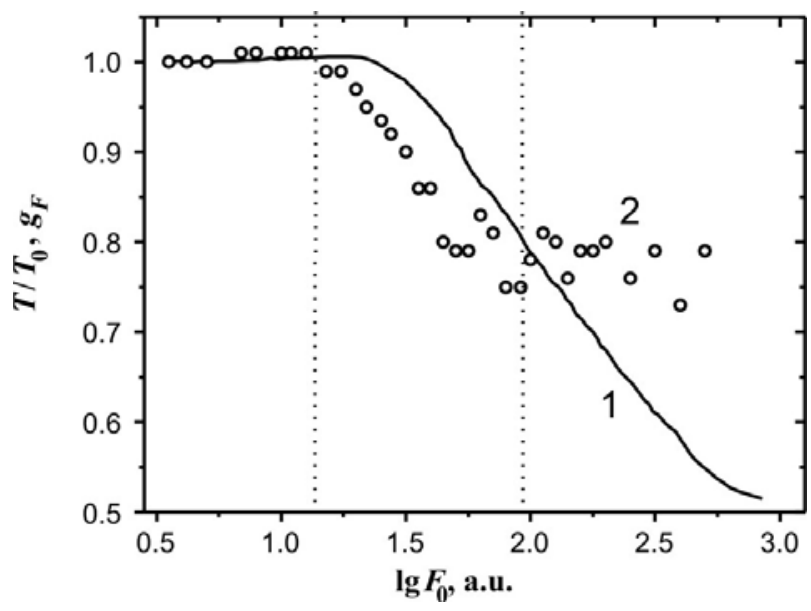

Fig. 4. Nonlinear transmittance curves of aqueous suspension of black-body particles for laser pulses $20 \mathrm{~ns}, 1064 \mathrm{~nm}$. Numbers near the curves represent concentrations of particles in relative units. 
The observed change from quick to slow decrease of $\gamma_{F}$ with the increase of $F_{0}$ (see Fig. 3) is not explained by the proposed simple $\chi^{(5)}$ model. It is plausible to suggest that the mentioned turn of $\gamma_{F}\left(F_{0}\right)$ curve indicates hidden changes in the physical processes responsible for observed optical limiting in the investigated suspensions.

Now consider another method of analysis of nonlinear transmittance curves. The equation (1) can be integrated along the laser track as follows

$$
\ln T=-\int_{0}^{d} \alpha(z) N d z
$$

The integral in (8) can be presented in the following form $\int_{0}^{N d} \alpha d(N z)$. Then, a derivative of (8) with respect to the parameter $N$ yields the following expression

$$
\alpha(F)=-\frac{1}{d} \cdot \frac{\Delta(\ln T)}{\Delta N}
$$

where the value of extinction cross section, $\alpha$, corresponds to the laser power density $F=F_{d}$. The expression (9) gives the intensity dependence of the extinction cross section, which can be obtained from the experimental data (nonlinear transmittance curves) measured using suspensions (or solutions) of different concentrations.

As an example of applying the expression (9), consider optical limiting in carbon black suspensions. A series of nonlinear transmittance curves measured with suspensions of different particle concentrations is given in Fig. 4. These data were treated according to (9), and the nonlinear part of the extinction cross section, $\alpha-\alpha_{0}$,

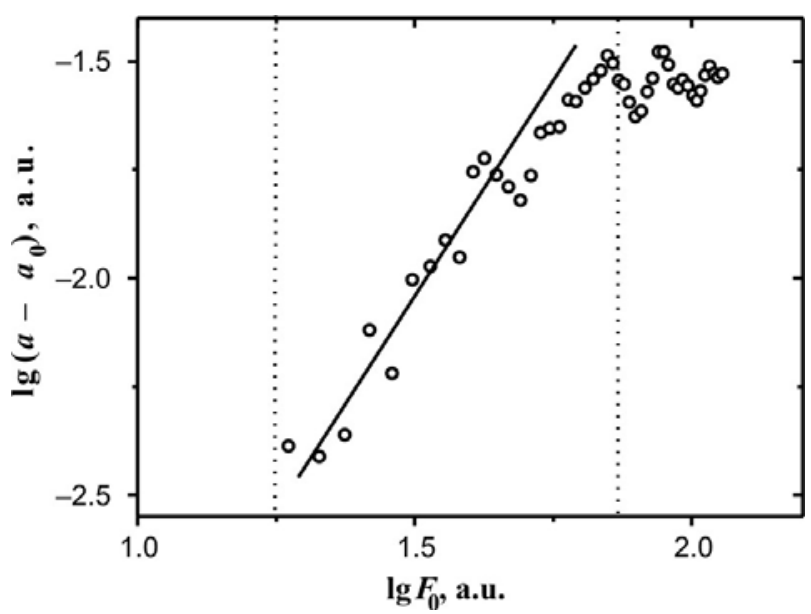

Fig. 5. Nonlinear part of extinction cross section as a function of laser power density (1064 nm, $20 \mathrm{~ns})$ in aqueous suspension of black-body particles. is plotted against $F_{0}$ in Fig. 5 in a log-log scale. The data depicted in Fig. 5 correspond to the region of the incident laser intensity, $1.25<\lg F_{0}<1.9$, where the $\gamma_{F}$ curve is located below the transmittance curve (within the margins marked by dashed lines in Fig. 3). A straight solid line in Fig. 5 is drawn with a slope of 2. As is seen, the slope of the plot in Fig. 5 is close to 2 (linear fit gives the value 1.7). This circumstance indicates that optical limiting in carbon suspensions is primarily determined by the fifth-order optical nonlinearities. Therefore, both of the considered methods of analysis of nonlinear transmittance curves lead to the similar conclusions.

\section{Concluding remarks}

In this paper, we performed a comparative study of optical limiting caused by excited state absorption and nonlinear scattering of $(10 \ldots 20) \mathrm{ns}$ laser pulses in crystals, glasses, and suspensions. The results obtained give us grounds to conclude that differences in the mechanisms of laser radiation interaction with matter manifest themselves in the differences of shape of nonlinear transmittance curves. To reveal the mentioned differences, we propose two simple methods based on differentiation of nonlinear transmittance curves. Using the formalism of nonlinear optical susceptibilities, we conclude that the proposed methods of analysis provide to reveal the fifth-order optical nonlinearities in the investigated processes.

\section{References}

1. P.G. Kriukov, V.S. Letokhov, Propagation of light pulses in media with resonant amplification (absorption) // Uspekhi fiziki 99(2), p. 169-227 (1969) (in Russian).

2. D.N. Klyshko, Physical principles of quantum electronics, Ed. A.A. Rukhadze, Nauka, Moscow (1986) (in Russian).

3. H.S. Kwok, T.M. Rossi, W.S. Lau, D.T. Shaw, Enhanced transmission in $\mathrm{CO}_{2}$-laser -aerosol interactions // Opt. Lett. 13(3), p. 192-194 (1988).

4. E.A. Tikhonov, M.T. Shpak, Nonlinear optical phenomena in organic compounds. Naukova Dumka, Kiev (1979) (in Russian).

5. V.S. Letokhov, Nonlinear selective photoprocesses in atoms and molecules. Nauka, Moscow (1983) (in Russian).

6. S.Venugopal Rao, N.K.M.Naga Srinivas, D.Narayana Rao, Nonlinear absorption and excited state dynamics in Rhodamine B studied using z-scan and degenerate four wave mixing techniques // Chem. Phys. Lett. 361, p. 439-445 (2002).

7. P. Sathy, R. Philip, V.P.N. Nampoori, C.P.G. Vallabhan, Photoacoustic observation of excited singlet state absorption in the laser dye 
Rhodamine 6G // J Phys. D: Appl.Phys. 27, p. 20192022 (1994)

8. C.B. de Araujo, G.S.Maciel, N.Rakov, Y.Messaddeq, Giant non-linear absorption in $\mathrm{Er}^{3+}$-doped fluoroindate glass // J. Non-Cryst. Solids 247(1-3), p. 209-214 (1999).

9. M.U. Bely, S.E. Zelensky, B.A. Okhrimenko, S.M. Yablochkov, Luminescence of mercury-like ions in glasses at powerful excitation // Izvestia AN SSSR. Ser. Phys. 49(10), p. 2010-2014 (1985)(in Russian).

10. M.U. Bely, O.S. Kolesnik, B.A. Okhrimenko, V.P. Yashchuk, Sequential transitions in thallium complexes // Sov. Appl. Spectr. Journ. 50(5), p. 808814 (1989).

11. S.E. Zelensky, Self-induced attenuation of pulsed laser radiation in an aqueous suspension of submicron light-absorbing particles // J. Phys.: Condens. Matter 15, p. 6647-6657 (2003).

12. K. Mansour, M.J. Soileau, E.W. van Stryland, Nonlinear optical properties of carbon-black suspensions (ink) // J. Opt. Soc. Amer. B 9(7), p. 1100-1109 (1992).

13. K.M. Nashold, D.P. Walter, Investigations of optical limiting mechanisms in carbon particle suspensions and fullerene solutions // Ibid. 12(7), p. 1228-1237 (1995).

14. S.K. Tiwari, M.P. Joshi, S. Nath, S.C. Mehendale, Salt-induced aggregation and enhanced optical limiting in carbon-black suspensions // J. Nonlinear Opt. Phys.\& Materials 12(3), p. 1-5 (2003).

15. S.K. Tiwari, M.P. Joshi, M. Laghate, S.C. Mehendale, Role of host liquid in optical limiting in ink suspensions // Optics \& Laser Technology 34, p. 487-491 (2002).

16. M.U. Bely, S.E. Zelensky, B.A. Okhrimenko, S.M. Yablochkov, Interaction of $\mathrm{Tl}^{+}$centers in potassium-borate glass with powerful laser radiation // Ukr. Fiz. Zhurn. 27(7), p. 1002-1006 (1982) (in Russian).

17. S.E. Zelensky, Non-uniformity of crossbeam laser power distribution as a source of errors in non-linear spectroscopy // Semiconductor Physics, Quantum Electronics and Optoelectronics 6(3), p. 378-381 (2003).

18. M.U. Bely, S.E. Zelensky, B.A. Okhrimenko, On the errors in luminescent analysis caused by the violation of a preset field approximation // Ukr. Fiz. Zhurn. 37(7), p. 994-998 (1992) (in Russian).

19. A.A. Kaminskii, Modern developments in the physics of crystalline laser materials // Phys. status solidi (a) 200(2), p. 215-296 (2003).

20. R.T. Wegh, A. Meijerink, R.-J. Lamminmaki, J. Holsa, Extending Dieke's diagram // J. Luminescence 8789, p. 1002-1004 (2000).

21. G.J. Quarles, G.E. Venikouas, R.C. Powell, Sequential two-photon excitation processes in $\mathrm{Nd}^{3+}$ ions in solids // Phys. Rev. B 31(11), p. 6935-6940 (1985).

22. M.U. Bely, S.E. Zelensky, B.A. Okhrimenko, S.M. Yablochkov, Effect of laser radiation on absorption and luminescence of tin-doped potassiumborate glass // Ukr. Fiz. Zhurn. 29(2), p. 175-178 (1984).

23. M.U. Bely, S.E. Zelensky, B.A. Okhrimenko, S.M. Yablochkov, Interaction of luminescence centers in potassium-borate glass with powerful laser radiation // Optics and Spectroscopy 55(1), p. 78-82 (1983).

24. O.V. Kopyshinsky, B.A. Okhrimenko, S.E. Zelensky et al. Laser- and gamma-induced transformations of optical spectra of indium-doped sodium-borate glass // J. Phys.: Condens. Matter 15, p. 3919-3929 (2003).

25. S.E. Zelensky, Laser-induced non-linear light scattering in a suspension of black-body particles // Semiconductor Physics, Quantum Electronics and Optoelectronics 7(2), p. 190-194 (2004).

26. K.J. McEwan, P.A. Madden, Transient grating effects in absorbing colloidal suspensions // J . Chem. Phys. 97(11), p. 8748-8759 (1992).

27. X. Sun, Y. Xiong, P. Chen, et al. Investigation of an optical limiting mechanism in multiwalled carbon nanotubes // Appl. Opt. 39(12), p. 1998-2001 (2000).

28. X. Sun, R.Q. Yu, G.Q. Xu, et al. Broadband optical limiting with multiwalled carbon nanotubes // Appl. Phys. Lett. 73(25), p. 3632-3634 (1998). 